\title{
Application of Intermediate Length Catheter in Palliative Treatment of Tumor
}

\author{
Hengyu Cai ${ }^{1}$, Qi Jiang ${ }^{2, *}$, Qun Lou ${ }^{2}$, Yixuan Wang ${ }^{2}$, Hongyan Guo ${ }^{1}$ \\ ${ }^{1}$ Peparterment of Hematology Oncology,China-Japan Union Hospital of Jilin University, Changchun, China \\ ${ }^{2}$ Department of Nephrology, China-Japan Union Hospital of Jilin University, Changchun, China
}

\section{Email address:}

710715634@qq.com (Qi Jiang)

${ }^{*}$ Corresponding author

\section{To cite this article:}

Hengyu Cai, Qi Jiang, Qun Lou, Yixuan Wang, Hongyan Guo. Application of Intermediate Length Catheter in Palliative Treatment of Tumor. Science Discovery. Vol. 7, No. 1, 2019, pp. 9-12. doi: 10.11648/j.sd.20190701.12

Received: January 17, 2019; Accepted: February 21, 2019; Published: March 8, 2019

\begin{abstract}
Today's society,tumors have become the number one killer treateningt the well-being of human beings.At present,China swollen and persistent disease rises every year.Bring a heavy burden on family and society. Although the treatment is becoming more and more popular,it is still the main cause of death and chronic colouring disease.In particular,it is becoming more and more important for the clinicians to pay attention to the fact that it is impossible to cure the disease,so the palliative treatment can lead to a high quality of life in the final stage of their life.In Auntie, pulse-sparing infusion is still the main mode of administrain.In the course of platinum solution treatment,repaeted puncture not only increased the patient's pain, but also occurred with plstinum fluid. The medium length of the guide was chosen, Procedures for operation, not only less puncture and blowing number, through the patient pain, complications also rare.The goal of Treatment of Patients with Swelling and Peeding Benefits obtained sood results.
\end{abstract}

Keywords: Intermediate Length Catheter, Palliative Treatment of Tumor, Not for Treatmeat, Complication

\section{中等长度导管在肿瘤姑息治疗中的应用}

${\text { 蔡街玉 }{ }^{1} \text {, 姜琦 }{ }^{2 *} \text {, 娄群 }{ }^{2}, \text { 王艺璇 }{ }^{2} \text {, 郭鸿延 }}^{1}$

1吉林大学中日联谊医院肿瘤血液科, 长春, 中国

2吉林大学中日联谊医院肾病内科, 长春, 中国

邮箱

710715634@qq.com（姜琦）

摘要：当今社会, 肿瘤已成为威胁人类健康的头号杀手。目前, 我国肿瘤发病率逐年上升, 给家庭及社会都带来了沉 重的负担[1-3]。虽然治疗手段越来越多, 但其仍成为主要致死率的慢性疾病。尤其肿瘤晚期病人在不可能治愈疾病时, 姑息治疗可在其生命最后阶段提高生活质量, 因此已越来越被临床所重视[4]。在姑息治疗中, 静脉输注仍为主要给药 方式。而输液治疗过程中, 反复穿刺不仅增加病人痛苦, 输液并发症也随之出现[5]。而选择中等长度导管, 进行程序 化操作, 不但减少穿刺次数, 减轻病人痛苦, 并发症也少有发生, 最终达到提高患者生活质量的目标[6-7]。我科通过 对肿瘤患者进行姑息治疗采用中等长度导管输液取得了良好效果。

关键词: 中等长度导管, 肿瘤, 姑息治疗, 并发症 


\section{1. 资料与方法}

\section{1. 一般资料}

选择我科2017年9月至2018年10月收治的180例肿瘤 姑息治疗患者作为研究对象。纳入标准:(1)预输液时间大 于等于一周; (2) 血常规及凝血功能正常。排除标准:

（1）置管部位有放射史、血栓史及外伤史; （2）插管部 位有感染史或对导管有过敏史; (3) 置管前3天有发热史。 将患者随机分为观察组90例和对照组90例。其中男98例, 女 82 例; 年龄 $15 \sim 85$ 岁, 平均 $(65.4 \pm 2.3$ 岁); 肺癌42例, 肠癌36例, 胃癌28例, 乳腺癌32例, 脑肿瘤6例, 骨肿瘤 10例, 食管癌4例, 胰腺癌3例, 淋巴瘤 12 例, 转移癌7例。 两组患者在年龄、性别及病情等方面比例, 差异无统计学 意义 $(P>0.05)$ 具有可比性。

\section{2. 方法}

\subsection{1. 对照组}

采用外周静脉留置针 $22 \sim 24 \mathrm{G}$, 穿刺部位为外周浅静 脉, 留置时间为 $24 \sim 72$ 小时。

\subsection{2. 观察组}

采用外周穿刺置入中等长度导管。属于外周静脉导管 因此不需X线进行尖端定位。于 19 世纪 50 年代开始应用于 临床[8-10], 留置时间按推荐为 $2 \sim 6$ 周 [11]。

\section{A. 材料}

选用 $4 \mathrm{Fr}$ 单腔一次性使用经外周静脉置入中心静脉导 管套件（法国美德），聚氨酯材料长度 $40 \mathrm{~cm}$ 。

\section{B. 置管操作}

(1)置管人员由具有PICC 证书的专科护士操作。(2) 血管选择肘部首选贵要静脉, 其次是肘正中静脉及头静脉, 颈部选择颈外静脉。（3）置管长度：外周静脉一中等长 度导管尖端位置在腋窝水平 [12], 一般长度为 $18 \sim 25 \mathrm{~cm}$, 测量方法: 手臂外展90度, 测量从穿刺点至肩锁关节长度。 而颈外静脉根据分型不同, 前端位置在颈内静脉或锁骨下 静脉, 长度约为 $8 \sim 12 \mathrm{~cm}$ 。（4）用物准备：穿刺包、生理 盐水、肝素钠、无菌手套、一次性换药包（碘伏棉球、酒 精棉球、镊子 2 把、弯盘 2 个) 、正压接头。(5)皮肤消毒: 以穿刺点为中心, 三遍酒精、三遍碘伏消毒、待干。肘部 消毒面积为整个手臂。颈部消毒面积以穿刺点为中心上至 同侧下领骨，下至同侧锁骨下缘，左右至 $10 \mathrm{~cm}$ 。再用无菌 治疗巾建立最大无菌屏障。（6）静脉穿刺：先用利多卡 因湿敷穿刺点 $5 \sim 10$ 分钟, 以减轻穿刺时的疼痛感。穿刺 时采用改良塞丁格技术, 既可以提高穿刺成功率, 也可以 减少并发症的发生率。穿刺成功后, 送入导丝 $10 \mathrm{~cm}$ 左右即 可、退穿刺针、用 $1 \mathrm{ml}$ 注射器皮下注射利多卡因进行局部 麻醉、沿导丝在皮肤上方用手术刀进行切开、插入扩皮器 和可撕裂鞘、撤导丝及扩皮器, 从撕裂鞘送入导管达到预 期长度。连接正压接头, 冲管、封管, 无菌敷料固定导管。 肘部穿刺体位采用平卧位, 置管侧肢体外展 $90^{\circ}$, 头偏向
对侧。颈外静脉穿刺体位采用去枕平卧, 头低足高位, 头 偏向对侧, 充分暴露穿刺静脉; 静脉显示不清者, 可由助 手按压胸锁乳突肌, 使静脉充盈。

\section{3. 维护方法}

\subsection{1. 对照组}

每日观察穿刺部位局部皮肤情况: 有无红肿、疼痛; 沿静脉走行有无条索状改变; 贴膜是否完整、松脱。若出 现药液渗出、静脉炎、液体输入不畅时要拔除静脉留置针。 每日输液前用 $3 \sim 5 \mathrm{ml}$ 生理盐水冲管, 再静脉给药; 输液结 束后用生理盐水 $5 \mathrm{ml}$ 正压封管。当贴膜松脱、潮湿, 穿刺 点出血、渗液时要及时更换敷贴。

\subsection{2. 观察组}

护理方法同PICC维护方法。

\section{A. 穿刺部位局部护理}

穿刺成功后避开穿刺点, 沿静脉走行外贴水胶体敷料。 置管后24小时更换敷料, 注意穿刺点渗血情况。在导管使 用过程中, 每周更换无菌敷料及正压接头。若患者出汗多、 有渗血或渗液, 敷贴潮湿、有卷边等情况要随时更换。

\section{B. 并发症护理}

中等长度导管的并发症与PICC 导管相似, 包括静脉炎、 血栓、导管脱出、导管相关性感染和导管堵塞等。置管前 三日, 每 $4 \sim 6$ 小时湿热敷穿刺点及导管所经的静脉, 以促 进血液循环, 预防血栓及静脉炎的发生。输液时抬高肢体, 多做握拳运动, 促进静脉回流, 起到预防静脉炎的效果。 护士严密观察输液侧肢体有无肿胀、疼痛, 测量臂围增加 $2 \mathrm{~cm}$ 以上时, 要及时行血管超声检查排查血栓的发生。合 理安排输液顺序, 输注白蛋白、静脉营养液等粘稠液体后 要及时用生理盐水 $20 \mathrm{ml}$ 脉冲式冲管; 避免药物相互作用, 有配伍禁忌的药物间要冲管, 避免导管堵塞。封管液选择 $10 \mathrm{IU} / \mathrm{ml}$ 的肝素稀释液。输液前用酒精棉片消毒正压接头 的正面及侧面, 摩擦消毒 $15 \sim 30$ 秒, 待干。正压接头每7 天更换一次, 但接头中有血液、接头明确被污染或接头被 移除后需要重新更换[13]。

\section{4. 观察指标}

主要对两组置管并发症的发生率及患者满意度进行 观察统计。

\section{5. 统计学处理}

应用SPSS17.0统计软件进行数据分析, 计数资料以率 或构成比表示, 采用 $\mathrm{X}^{2}$ 检验; $\mathrm{P}<0.05$ 为差异有统计学意 义。

\section{2. 结果}

观察两组并发症的发生率及患者满意度情况差异。对 照组发生静脉炎13例, 药液渗出9例, 静脉血栓1例, 导管 
堵塞 2 例, 相关感染 1 例; 观察组发生静脉炎 2 例, 药液渗 出 0 例, 静脉血栓 1 例, 导管堵塞 1 例, 导管相关感染 1 例。 中等长度导管并发症的发生率明显低于外周留置针组, 差
异有统计学意义 $(\mathrm{P}<0.05)$ 。同时, 观察组患者满意度 高于对照组, $\mathrm{P}<0.05$ 。详见表1及表2。

表1 两组患者合并症发生率比较。

\begin{tabular}{lllllll}
\hline 分组 & 例数 & 静脉炎 & 药液渗出 & 导管相关感染 & 导管堵塞 & 血栓 \\
\hline 实验组 & 90 & 2 & 0 & 1 & 1 & 1 \\
对照组 & 90 & 13 & 9 & 1 & 2 & 1 \\
\hline
\end{tabular}

$\mathrm{X}^{2}=17.2, \quad \mathrm{P}<0.01$

表2 两组患者满意度情况比较。

\begin{tabular}{llll}
\hline 分组 & 例数 & 满意 & 不满意 \\
\hline 实验组 & 90 & 84 & 6 \\
对照组 & 90 & 75 & 15 \\
\hline
\end{tabular}

$\mathrm{X}^{2}=4.37, \quad \mathrm{P}<0.05$

\section{3. 讨论}

\section{1. 姑息治疗的临床意义}

姑息治疗是人性化治疗的一种新理念, 肿瘤患者进行 姑息治疗的目的在于提高生存质量, 使其在生命最后阶段 享受相对舒适的生活，因此在临床上得到广泛应用 [14]。 而在姑息治疗中, 静脉输注仍是主要方式之一; 多数病人 前期反复用药, 或疾病晚期不能进食累及外周循环差, 导 致外周静脉壁越来越硬、管腔越来越窄、弹性越来越差 [15]。 使用套管针, 需要反复穿刺, 这不仅增加病人痛苦, 同时 药液外渗也给病人及护士带来风险, 这与姑息治疗的理念 相违背[16]。

\section{2. 中等长度导管在姑息治疗中的优势}

\subsection{1. 中等长度导管的适应症}

2011年美国输液护士协会（INS）静脉输液治疗护 理实践标准推荐 [17]: 所有能经外周浅静脉装置的药物 和液体均适用于中等长度导管, 不适合输注的药物和液 体包括持续腐蚀性药物治疗、胃肠外营养、 $\mathrm{pH}$ 值低于5 或高于 9 的液体或渗透压大于 $600 \mathrm{mosm} / \mathrm{L}$ 的液体。然而 欧洲临床营养和代谢学会 (ESPEN) 指南指出: 由于外 周浅静脉发生移位和渗出的风险高, 对于居家需要行 PPN的病人, 中等长度导管更适合 [18]。本案例中进行 肠胃营养的患者, 采用中等长度导管组无 1 例出现药液 渗出, 与ESPEN指南相吻合。而外周留置针组, 肠外营 养的患者出现7例。而且Caparas等[19]做的前瞻性研究 得出: 经中等长度导管静脉输注万古霉素 $(\mathrm{ph}=3.9)$ 是 安全可行的。我国近几年的文献[20]中显示：过敏性紫 癜、神经内科病人、血液科肿瘤病人静脉输液治疗, 使 用中等长度导管均取得良好效果。肿瘤姑息治疗患者静 脉输注的安全性既是治疗的前提, 又是提高其生存质量 的保证, 而合理选择输液工具的类型是保障安全输液的 重要因素。综上所述, 肿瘤患者姑息治疗采用中等长度 导管是安全、合理、可行的。

\subsection{2. 中等长度导管的优点}

中等长度导管置管操作简单, 由护士单独操作即可 [21]。PICC留置时间虽长, 但费用较高。中心静脉置管留 置时间与中等长度导管相近, 但穿刺风险大, 并发症高 [22-23]。2011版《血管内导管相关感染的预防指南》中指 出: 中等长度导管与外周短导管相比, 可以降低静脉炎的 发生率; 而与中心静脉导管相比, 可以降低血流感染率[24]。 因为中等长度导管尖端位于腋静脉, 血液流速为 $100 \mathrm{ml} / \mathrm{min} \sim 150 \mathrm{ml} / \mathrm{min}$, 高于外周浅静脉 $20 \mathrm{ml} / \mathrm{min} \sim$ $40 \mathrm{ml} / \mathrm{min}$, 较快的血流速度可以快速稀释药液浓度, 从而 减少药物对血管内皮的损伤, 降低化学性静脉炎的发生 [25]。本案例中观察组发生静脉2例, 而对照组发生13例, 结果与指南相契合。

与外周留置针相比, 中等长度导管除并发症少外, 病 人的舒适度也大幅度增加。因为外周留置针在关节或近关 节处, 活动受限, 病人会不自觉地制动穿刺侧肢体, 影响 其舒适度; 若不经意活动, 导管也容易脱出或打折, 而影 响其使用。而且对中等长度导管病人维护及宣教过程中, 可以更贴近病人, 倾听患者的不适、解答病人的疑问, 给 与病人适度的关怀。因此, 两组病人对护士满意度情况对 比存在显著差异, 有统计学意义 $(\mathrm{P}<0.05)$ 。

中等长度导管尖端位于大静脉而不是中心静脉, 因此 不需要放射线确认前端位置[26]。这对于肿瘤姑息治疗的 患者来说, 不仅节约医疗成本, 也避免了患者因拍片带来 的不便, 尤其是行动受限病人。这从某种意义上讲, 提高 了肿瘤姑息病人的生活质量[27]。

\section{3. 中等长度导管在肿瘤姑息治疗的局限性}

中等长度导管留置时间按推荐为 $2 \sim 6$ 周, 对于肿瘤姑 息治疗中的部分患者, 如: 年龄 $<50$ 岁, 无其他基础疾病, 对药物敏感、姑息治疗效果好等病人来讲, $2 \sim 6$ 周时间相 对短, 涉及拔除导管或置换新导管, 但这对病人来讲无疑 是不利的。而在没有并发症的情况下, 是否可以适当延长 使用时间, 是有待进一步研究探讨的。

肿瘤姑息治疗中, 静脉营养和脱水治疗是较常见的, 常见脱水剂, 如甘露醇的渗透压为 $1098 \mathrm{mosm} / \mathrm{L}$, 而静 脉营养液渗透压高达 $1100 \sim 1400 \mathrm{mosm} / \mathrm{L}$, 高渗透压药 物在指南中是不适用于中等长度导管的。本案例中虽未 出现相应药液外渗, 但强酸强碱药物、高渗透压药物的 使用频率、使用方法与相关并发症之间的关系有待进一 步研究。 


\section{4. 结论}

中等长度导管位于外周静脉, 无需进行导管尖端定位, 可作为外周静脉留置针和PICC之间的一座桥梁, 多位学者 研究表明对于外周静脉条件差、预期输液时间较长的患者 早期使用中等长度导管可减少反复穿刺、减轻患者痛苦、 提高患者的生活质量, 降低了经济成本, 增加经济效益。 是一种安全可靠的输液工具。在肿瘤姑息治疗中, 与外周 留置针相比, 输液并发症少, 患者满意度高, 能提高患者 的生存质量, 值得临床推广使用。因此, 建议相关机构在 静脉输液培训中增加中等长度导管的知识, 加大中等长度 导管的研究和使用力度, 进一步界定中等长度导管的留置 时间、适应症及禁忌症, 旨在为患者选择更为舒适、安全 的静脉输液通路。

\section{参考文献}

[1] 杜风华, 闵旭红, 梅晓冬. 肺腺癌靶向治疗药物的应用及 耐药机制 [J].临床肺科杂志, 2018,23(01): 168-172。

[2] 张秋杰,邱文生.恶性肿瘤姑息治疗的现状与展望 [J].齐鲁医 学杂志,2017,32(01):124-126。

[3] 宋纯杰.姑息关怀对肿瘤病人自杀倾向的影响与干预[J].全 科护理,2018,16(12):1447-1449。

[4] 陈华俊, 彭娜.PICC置管用于肿瘤姑息治疗的效果观察 [J]. 现代医药卫生，2015,31（13）：2040-2041。

[5] 陈海燕,欧社娣,潘珊,黄晓倩,郭月,谢秀中,胡洁琴,刘红利.改 良中等长度PICC 导管在老年慢性阻塞性肺疾病患者中的 应用[J].广东医学,2018,39(13):2048-2049。

[6] 胡明明,沈小芳,顾平,陈湘玉.外周静脉中等长度导管的临床 应用研究现状[J].护理研究,2015,29(31):3845-3848。

[7] 肖沙璐.外周静脉中等长度导管尖端位置与相关并发症的 研究现况与展望[A].上海市护理学会.第三届上海国际护理 大会论文摘要汇编 $[C]$. 上海市护理学会: 上海市护理学 会,2017:1。

[8] Sharp R, Esterman A, McCutcheon H, et al. The safety and efficacy of midlines compared to peripherally inserted central catheters for adult cystic fibrosis patients: A retrospective, observational study [J]. International Journal of Nursing Studies, 2014, 51(5): 694-702.

[9] 陶新曹, 刘国梁, 申艳玲, 等. 重症患者静脉输液通路选 择策略的调查研究 $[\mathrm{J}]$. 中国呼吸与危重监护杂志, 2016, 15(1):57-59。

[10] 李成涛, 张敏, 赵䒯. 单体结构对酶促合成脂肪族聚酯聚 合度的影响 $[\mathrm{J}]$. 陕西科技大学学报, 2017, 35(5):117121 。
[11] Infusion Nurses Society. Infusion Nursing Standards of Practice [J]. J Infus Nurs, 2006, 29(1 suppl): S1-S2.

[12] 中华护理学会静脉输液治疗护理专业委员会编译.(2011)输 液治疗护理实践标准[J].输液治疗护理杂志, 2011,34(1S): $33-40$ 。

[13] 王建荣.输液静脉治疗护理实践指南与实施细则 $[\mathrm{M}]$. 北京: 人民军医出版社, 2011：S29。

[14] 蔡丽华, 李健秋, 赵萍.外周中心静脉置管在肿瘤化疗患者 中的应用体会 [J]. 实用临床医药杂志, 2013, 17 (4): 13-15。

[15] 王玉华, 周小伟.肿瘤患者外周静脉置管术后感染因素分析 及预防对策 [J]. 中华医院感染学杂志, 2013,23（8）: 1790-1791。

[16] 王敏, 贾玉霞.探讨PICC在肿瘤护理中的临床应用分析 [J]. 中国保健营养，2013（2）：8。

[17] Gorski LA, Eddins J,Hadaway L,et al. Infusion nusing standards of practice[J].J Infus Nurs,2013,34(1S):S37-S38.

[18] 胡明明, 沈小芳, 顾平, 等.外周静脉中等长度导管的临床 应用研究现状 [J].护理研究2015,29（11）:3845-3848。

[19] Caparas J V, Hu J P. Safe Administration of Vancomycin through a Novel Midline Catheter.A Randomized, Prospective Clinical Trial [J]. J Vasc Access, 2014,15(4):251-256.

[20] 刘艺红, 刘燕, 邱晓燕, 等.中等长度导管在神经内科病人 中的应用及常见问题分析 [J]. 护理实践与研究, 2009,6 (20) :102-103。

[21] 刘佳佳,张娟,谭蕾.改良型中等长度导管与PICC在胃肠道肿 瘤患者围手术期中的应用比较 [J]. 护理实践与研 究,2018,15(07):143-145。

[22] Adams DZ,Little A,Vinsant C,et al. The Midline Catheter:A Clinical Review [J]. J Emerg Med,2016,51(3):252 - 258.

[23] 陶新曹, 刘国梁, 申艳玲, 等. 重症患者静脉输液通路选 择策略的调查研究 $[\mathrm{J}]$. 中国呼吸与危重监护杂志, 2016, 15(1):57-59。

[24] O'Grady N P, Alexander M, Buerns L A,et al. Guidelines for the Pre-Vention of Intravascular Catheter-related Infections [J]. Am J Infect Control, 2011, 39(4Supp11):1-34.DOI:10.1016/j.ajic.2011.01.003.

[25] 杨莘, 花蕾, 邵文利, 等.静脉输液护理指南[M].北京: 科 学技术文献出版社, 2009:45-46。

[26] Anderson NR.Middle catheters:The middle ground of intravenous therapy administration [J]. J Infus Nurse, 2004, 27:313-321.

[27] 王亚琳.中长导管静脉置管在急诊临床的应用现状和展望 [J].大医生,2018,3(06):138-139。 